\title{
A fronto-parietal circuit for object manipulation in man: evidence from an fMRI-study
}

\author{
F. Binkofski, ${ }^{1}$ G. Buccino, ${ }^{2}$ S. Posse,${ }^{3}$ R. J. Seitz, ${ }^{1}$ G. Rizzolatti, ${ }^{2}$ H.-J. Freund ${ }^{1}$ \\ ${ }^{1}$ Department of Neurology, Heinrich-Heine-University of Düsseldorf, Moorenstrasse 5, D-40225 Düsseldorf, Germany \\ ${ }^{2}$ Institute of Human Physiology, University of Parma, Italy \\ ${ }^{3}$ Institute of Medicine, Research Center Jülich GmbH, Germany
}

Keywords: inferior parietal cortex, object manipulation, secondary somatosensory area (SII), ventral premotor cortex

\begin{abstract}
Functional magnetic resonance imaging (fMRI) was used to localize brain areas active during manipulation of complex objects. In one experiment subjects were required to manipulate complex objects for exploring their macrogeometric features as compared to manipulation of a simple smooth object (a sphere). In a second experiment subjects were asked to manipulate complex objects and to silently name them upon recognition as compared to manipulation of complex not recognizable objects without covert naming. Manipulation of complex objects resulted in an activation of ventral premotor cortex [Brodmann's area (BA) 44], of a region in the intraparietal sulcus (most probably corresponding to the anterior intraparietal area in the monkey), of area SII and of a sector of the superior parietal lobule. When the objects were covertly named additional activations were found in the opercular part of BA 44 and in the pars triangularis of the inferior frontal gyrus (BA 45). We suggest that a fronto-parietal circuit for manipulation of objects exists in humans and involves basically the same areas as in the monkey. It is proposed that area SII analyses the intrinsic object characteristics whilst the superior parietal lobule is related to kinaesthesia.
\end{abstract}

\section{Introduction}

The capacity to grasp and manipulate objects is one of the hallmarks of motor dexterity in humans. It is lacking in prosimians and does not reach the degree of human precision and sophistication in apes. Effective grasping and object manipulation are based on three fundamental properties of the motor system: the capacity to generate independent finger movements, the ability to transform sensory information concerning the object to be grasped into an appropriate hand configuration, and a sophisticated somatosensory control of finger movements (see Jeannerod etal., 1995).

While it has been known since the thirties that independent finger movements depend on direct connections of the corticospinal tract with the spinal cord motoneurons (see Porter \& Lemon, 1993), the mechanisms through which sensory information controls grasping and manipulatory movements began only recently to be understood.

Recording and intracortical microstimulation studies showed that in the macaque monkey there is a large distal hand movements representation in the rostralmost part of ventral premotor cortex (area F5) (Rizzolatti etal., 1981, 1988; Kurata \& Tanji, 1986; HeppRaymond etal., 1994). The neurons of this area discharge during specific goal-directed hand movements such as grasping, holding and tearing. Many of them become active also in response to visual presentation of 3-D objects provided that these are congruent with the type of prehension coded by the recorded neuron (Rizzolatti et al., 1988; Murata et al., 1997). Area F5 is directly connected with the primary motor cortex $(\mathrm{F} 1)$ and receives rich input from the second somatosensory area (SII), from parietal area PF (7b), and from a

Correspondence: Dr F. Binkofski, as above.

Email: binkofsk@uni-duesseldorf.de

Received 12 November 1998, revised 28 April 1999, accepted 7 May 1999 parietal area located inside the intraparietal sulcus, the anterior intraparietal area (AIP) (Matsumura \& Kubota, 1979; Muakkassa \& Strick, 1979; Godschalk et al., 1984; Matelli etal., 1986; Luppino etal., 1999). The study of AIP showed that many of its neurons discharge during finger and hand movements, others respond to specific visual 3-D stimuli and, finally, others discharge both during active finger movements and in response to 3-D stimuli congruent in size and shape with the coded grasping movement (Taira et al., 1990; Sakata et al., 1992). Taken together, these data suggest that F5 plays a pivotal role in controlling the organization of hand-object interaction.

Brain imaging experiments carried out in humans failed up to now to convincingly demonstrate the existence of a cortical circuit similar to that described in the monkey. Using positron emission tomography (PET) techniques neither Rizzolatti et al. (1996) nor Grafton et al. (1996a) found any area specifically active during grasping movements. A blood flow increase in the premotor cortex was found only dorsally at the level of the superior frontal sulcus. This dorsal site was found to be active in tasks involving arm movements without grasping (Colebatch etal., 1991; Deiber etal., 1991; Grafton etal., 1992; Fink etal., 1997; Seitz etal., 1997). Finally, no blood flow increase was found in correspondence of the intraparietal sulcus where the putative human area AIP should be located (Binkofski et al., 1998a).

Slightly more encouraging results were reported by Matsumura etal. (1996). As Grafton et al. (1996a), they also asked subjects to point or grasp cylinders of different size. In contrast with the latter authors, they found a blood flow increase in a ventral premotor site, located at the rostral border of Brodmann's area (BA) 44. The importance of these finding is, however, diminished by the fact that no activation was found in the parietal region around the intraparietal sulcus. The significance of the premotor activation remains therefore 
unclear. Finally, Faillenot et al. (1997), in an experiment in which grasping was contrasted with pointing as well as with object shape matching, found only an activation of the left inferior postcentral sulcus when grasping was contrasted with pointing. When grasping was compared with matching, a condition in which no movement was required, several areas related to the sensorimotor system were active (central gyrus, postcentral sulcus, mesial motor areas, cerebellum, parietal operculum), but none that may be considered specific for grasping movements.

These essentially negative findings could be due to several possibilities. There may be interspecies differences in the organization of ventral premotor and intraparietal cortex. For example the development of a motor speech area in humans may have dramatically changed the location of the human functional homologue of monkey area F5. Intersubject variability may have obscured the comparisons and reduced the probability of obtaining intersubject coregistration and statistical significance in this area. In favour of this view are the data of Schlaug et al. (1994) showing a clear activation of Broca's area in single subjects during accurate finger movements. Finally, the task used in all the above experiments may have not required sufficient behavioural demand to activate the area involved in hand-object interactions. In all of them the objects to be grasped were rather simple and, most importantly, the movements were shortlasting and made at intervals.

The aim of the present experiment was to re-address the problem of whether a specific circuit involved in hand-object interaction is present also in humans and, if so, where it is located. As a main task we used a continuous manipulation of three-dimensional complex objects, either recognizable or not recognizable by means of manipulation. In contrast with previous tasks, ours required continuous finger movements and a constant change in finger configurations. Because of these requirements, we supposed that this task should be more efficient than those previously employed for activating brain areas involved in hand-object interactions. Furthermore, functional magnetic resonance imaging (fMRI), rather than the PET technique, was used.

Our results show that during manipulation of complex objects there is an activation of BA 44, a region in the intraparietal sulcus, SII and a sector of the superior parietal lobule. We propose that the circuit formed by these areas is the human homologue of the monkey grasping/manipulation circuit including areas AIP and F5.

\section{Methods}

\section{Subjects}

Twelve right-handed male subjects, aged $25-35$ years, were studied. Right-handedness was assessed by the Oldfield inventory (Oldfield, 1971). Two experiments were carried out with six subjects per experiment. None of the subjects had a current or past history of neurological disorders and each was normal on neurological examination. The study was approved by the Ethic Committee of the Heinrich-Heine-University, Düsseldorf. All subjects gave written consent prior to the study.

\section{MRI-scanner and scanning sequences}

Functional magnetic resonance imaging of cerebral blood oxygen level-dependent signal changes was performed as described in detail elsewhere (Binkofski et al., 1998a). Magnetic resonance (MR) images were recorded on a 1.5 Tesla Siemens 'Vision' MRI system (SIEMENS Magnetom, Erlangen, Germany), using standard echo planar imaging and a standard radio frequency head coil for signal transmission and reception. Sixteen axial slice positions (slice thickness, $4 \mathrm{~mm}$; interslice gap, $0.1 \mathrm{~mm}$ ) were orientated in the anterior-posterior commissure plane covering the brain volume above the temporal pole. The following sequences were used: gradient echo planar imaging, sequence repetition time (TR), $3 \mathrm{~s}$; signal (echo)-gathering time (TE), $66 \mathrm{~ms}$; FOV, $200 \times 200 \mathrm{~mm}$ (FOV, field of view); matrix size, $64 \times 64$; in-plane resolution, $3.125 \times 3.125$. In addition, high-resolution anatomical images of the entire brain were obtained by using a strongly T1-weighted gradient echo sequence (fast low-angle shot), sequences: TR, $40 \mathrm{~ms}$; TE, $5 \mathrm{~ms}$ (flip angle, $\alpha=40^{\circ}$ ), one excitation per phase-encoding step, FOV , $25 \mathrm{~cm}$, matrix size, $256 \times 256,128$ sagittal slices with $1.25 \mathrm{~mm}$ single slice thickness.

\section{Data acquisition and image analysis}

Image analysis was performed on a SPARC II workstation (Sun Microsystems) using MATLAB (Mathworks Inc., Natiek, MA, USA) and statistical parametric mapping package SPM96 (Friston etal., 1994a,b; 1995b, 1997; Poline etal., 1995; Worsley \& Friston, 1995). First, the 50 volume images of each condition were automatically realigned to the tenth image to correct for head movements between scans (Friston etal., 1995b). Then the images were coregistered and transformed into a standard stereotactic space, using the intercommissural line as the reference plane for transformation. During the normalization, pixels were slightly smoothed with a Gaussian filter to achieve isotropic voxels of $4 \times 4 \mathrm{~mm}$ in the $x$ and y dimensions, with an interplanar distance of $4 \mathrm{~mm}$. Voxels that had values $>0.8$ of the mean volume in all the images were selected to restrict the analysis to intracranial regions. The effects of global (whole volume) activity and time were removed as confounds, using linear regression and sine/cosine functions (up to a maximum of 2.5 cycles per 50 scans). Removing the latter confounds corresponded to high-pass filtering of the time series to remove low frequency artifacts, which could arise due to aliased cardiorespiratory and other cyclical components.

The stereotactically-normalized fMRI time-series data of the subjects were analysed separately. The alternating periods of 'baseline' and 'activation' were modelled using a simple delayed box-car reference vector accounting for the delayed cerebral blood flow change after stimulus presentation. Significantly activated pixels were searched for by using the 'General Linear Model' approach for time-series data suggested by Friston and colleagues (Friston etal., 1994a,b; Friston, 1995a, 1997; Poline et al., 1995; Worsley \& Friston, 1995). Therefore we defined a design matrix comprising contrasts that tested for significant activations during hand manipulation in each condition separately (tests for simple main effects). Group activation maps were calculated by pooling the data for each condition across all subjects. Pixels were identified as significantly activated if they passed the highest threshold of $Z=3.09$ and belonged to a cluster of at least 10 activated pixels $(P<0.05$, corrected for multiple comparisons) (Friston etal., 1994b). The activated pixels surviving the procedure were superimposed on high-resolution MR scans of a standard brain (Montreal Neurological Institute) and on 'SPM brain projections'.

With the aid of published Talairach-coordinates (Talairach \& Tournoux, 1988; Roland \& Zilles, 1996) and prominent sulcal landmarks (precentral, central and postcentral sulci, etc.) clusters of activated voxels were assigned according to their centre of mass activity. In addition the Talairach coordinates of the ventral premotor foci were compared with the coordinates of cytoarchitectonicallydefined probability maps related to Brodmann's areas (BAs) 44 and 45 (Amunts etal., 1998, 1999). 


\section{Experiment 1}

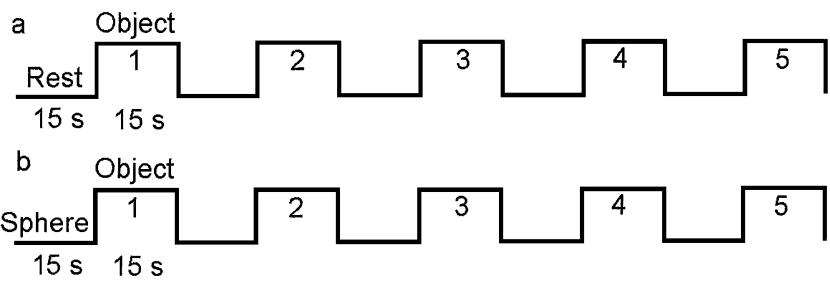

Experiment 2

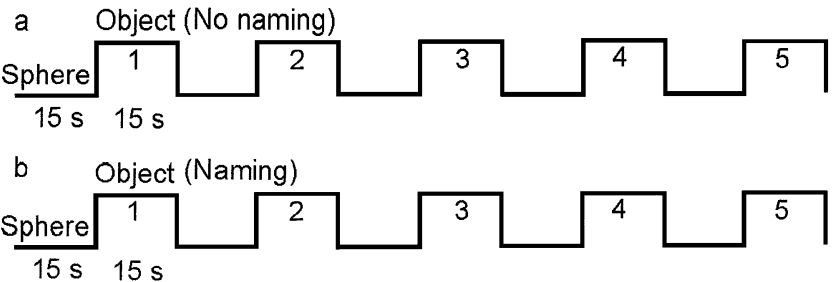

FIG. 1. A graphic representation of the experimental design used in the present experiments.

\section{Experimental protocols}

\section{Basic procedure}

Subjects lay supine in the scanner with the head immobilized with a pneumatic cushion and the eyes open. The room was dark. The subjects were asked to manipulate continuously either complex threedimensional objects or a sphere. Each object was placed into the subject's hand at the beginning of an activation phase and removed at the end of it by one of the experimenters. The object exchange was identical in all conditions. The subjects could not see the objects to be manipulated at any stage of the experimental procedure. Manipulation movements included movements of the thumb, index finger and the middle finger (see Kunesch etal., 1989; Seitz et al., 1991; Binkofski et al., 1992). Both hands were tested, separately. The testing order was randomized across subjects.

\section{Experiment 1}

The first experiment was designed to assess the cortical areas involved in manipulation of complex objects. It consisted of two experimental conditions for each of which five epochs of fMRI measurements were acquired. Each epoch was formed by a 15-s 'activation' phase, immediately followed by a 15-s 'baseline' phase (Fig. 1, upper part). On the whole 50 images for each condition were acquired. The total duration of one measurement was $2.5 \mathrm{~min}$. In the first condition (a) the activation phase consisted of a continuous manipulation of complex plastic objects, while the baseline phase consisted of rest, during which no motor activity was required. In the second condition (b) the activation phase was the same as in the first one, while the baseline consisted of continuous indifferent manipulation of a sphere. The condition order was randomized across the subjects. The objects to manipulate were small plastic toys of $\approx 3-$ $5 \mathrm{~cm}$ embodying real objects (e.g. houses, animals). During the activation phase of each epoch a different object to be manipulated was used. The subjects were asked to manipulate the objects carefully and to explore their basic features (surface, roughness, edges). Although the manipulated objects had a meaning and could therefore in principle be named by the subjects, preliminary tests excluded any of those used in experiment 1 that could be recognized by the subjects by manipulation. Subjects were informed that they were not required to recognize the objects located in their hands but only to manipulate them. The sphere that was manipulated during the baseline phase was made of plastic, had a smooth surface and a diameter of $3 \mathrm{~cm}$.

\section{Experiment 2}

As it will be shown in Results, experiment 1 demonstrated a strong activation of BA 44, an area involved in speech production. Considering this finding, a second experiment was designed the aim of which was to test whether the activation of BA 44, observed in the first experiment, was due to manipulatory finger movements or was related to an internal naming of object features. Like experiment 1 , experiment 2 consisted of two conditions. In the first condition (a) complex objects of similar material, size and surface characteristics as those of the first experiment were presented. The instruction was to manipulate the objects carefully, to explore their basic features (surface, roughness, edges) and to avoid any covert naming either of the objects or of their features. In the second condition (b) a set of common objects similar in material, size and surface characteristics as those used in the previous condition, but easy to recognize through manipulation (e.g. a matchbox, a small plastic car) was used. The instruction this time was to recognize the objects by means of manipulation and to name them covertly. At the end of the scanning session the subjects were asked to report the recognized objects. The experimental design of experiment 2 was the same as that of experiment 1. It is summarized in Fig. 1 (lower part).

\section{Results}

\section{Experiment 1}

The activations in this experiment and their anatomical locations are summarized in Table 1.

Manipulation of complex objects vs. rest activated the sensorimotor areas (primary somatosensory area, SI, and primary motor cortex, MI), the dorsal premotor cortex (dPMC) in the anterior bank of the precentral gyrus, the opercular part of the inferior frontal gyrus (ventral premotor cortex, vPMC), the supplementary motor area (SMA proper), the cingulate motor cortex (mCing, BA 24), the opercular parietal areas in the region corresponding to the secondary somatosensory area (SII), the superior parietal lobule (SP), and an area located in the anterior part of the lateral bank of the intraparietal sulcus. We refer to this last area as the anterior intraparietal area (AIP). SI, MI and mCing were activated contralateral to the manipulating hand whilst the $\mathrm{dPMC}$ and $\mathrm{vPMC}$ and the parietal areas AIP, SII and SP were activated bilaterally. An additional bilateral activation was observed in the inferior parietal lobule (IP) and some left-sided activity in the posterior part of the superior parietal lobule (PP) was observed during manipulation with the left hand. Some weak activations were found in the contralateral thalamus and in the posterior insula.

The comparison between the manipulation of complex objects and the manipulation of a sphere identified only a subset of areas activated in complex object manipulation vs. rest (Table 1). Among them the activated areas were: vPMC (BA 44), AIP, SII and SP and left IP. All these activations were bilateral. Weak additional ipsilateral activation was found in the inferior parietal lobule for both hands. For the left hand there was also a weak activation in the posterior part of the superior parietal lobule. The areas related to movement control, e.g. MI, SI, premotor areas, the SMA, and the thalamus, did not show up in this comparison.

\section{Experiment 2}

The results of this experiment are shown in Table 2 and Fig. 2. Figure 2 shows a general view of the activated areas from both 
TABLE 1. Functional areas significantly activated in the conditions of Experiment 1

\begin{tabular}{|c|c|c|c|c|c|c|c|}
\hline $\begin{array}{l}\text { Functional } \\
\text { area }\end{array}$ & \multicolumn{3}{|c|}{$\begin{array}{l}\text { Talairach } \\
\text { Coordinates }\end{array}$} & \multicolumn{2}{|c|}{ Complex object manipulation vs. rest } & \multicolumn{2}{|c|}{ Complex object manipulation vs. sphere manipulation } \\
\hline \multirow{2}{*}{ dPMC $r$} & 32 & -10 & 52 & 4.82 & 4.83 & & \\
\hline & 44 & -16, & 52 & 6.08 & & & \\
\hline vPMC r & 52 , & 8, & 20 & 5.10 & 4.99 & 3.52 & 3.6 \\
\hline Thal $\mathrm{r}$ & 12 & -16 & 4 & 3.31 & & & \\
\hline pIPS r & 40 & -48 & 50 & & & 4.06 & 4.83 \\
\hline IP r & 56 & -32, & 36 & 7.28 & 8.09 & 3.2 & 3.81 \\
\hline SP r & 36 & -52, & 60 & 4.98 & 6.36 & & 3.05 \\
\hline CING & 0 & 12, & 28 & 3.88 & 6.46 & & \\
\hline \multirow[t]{2}{*}{ SMA } & 4 & -12 & 64 & 3.69 & 3.89 & & \\
\hline & 4 & 0 & 44 & 6.73 & 6.21 & 5.92 & 5.3 \\
\hline M1/S1 1 & -46 & -32, & 50 & 6.78 & & & \\
\hline & 4 & 0 & 44 & 6.73 & 6.21 & 5.92 & 5.3 \\
\hline SII 1 & -64 & -20 & 24 & 7.59 & 7.5 & 5.86 & 5.93 \\
\hline AIP 1 & -40 & -40 & 40 & 7.82 & 7 & 6.82 & 6.14 \\
\hline pIPS 1 & -40 & -52 & 44 & 5.04 & 4.9 & 3.93 & \\
\hline IP 1 & -52 & -32 & 36 & 7.86 & 6.62 & & \\
\hline SP 1 & -32 & -56 & 56 & 5.25 & 4.6 & 3.93 & 3.88 \\
\hline PP 1 (G. ang.) & -16 & -76 & 52 & & 3.71 & & \\
\hline
\end{tabular}

Z-scores are presented, with the premotor activation foci fitting into the probability maps of BA 44 in bold. Abbreviations: 1, left; r, right; MI/SI; primary sensorimotor area; dPMC, dorsal premotor area; vPMC, ventral premotor area; Thal, thalamus; mCing, motor cingulate; SMA, supplementary motor area; SII, secondary somatosensory area; AIP, anterior intraparietal area; pIPS, posterior intraparietal; IP, inferior parietal lobule; SP, superior parietal lobule; PP, posteiror parietal area; G. ang., angular gyrus.

TABLE 2. Functional areas significantly activated with and without naming in Experiment 2, with manipulation of complex objects vs. spheres

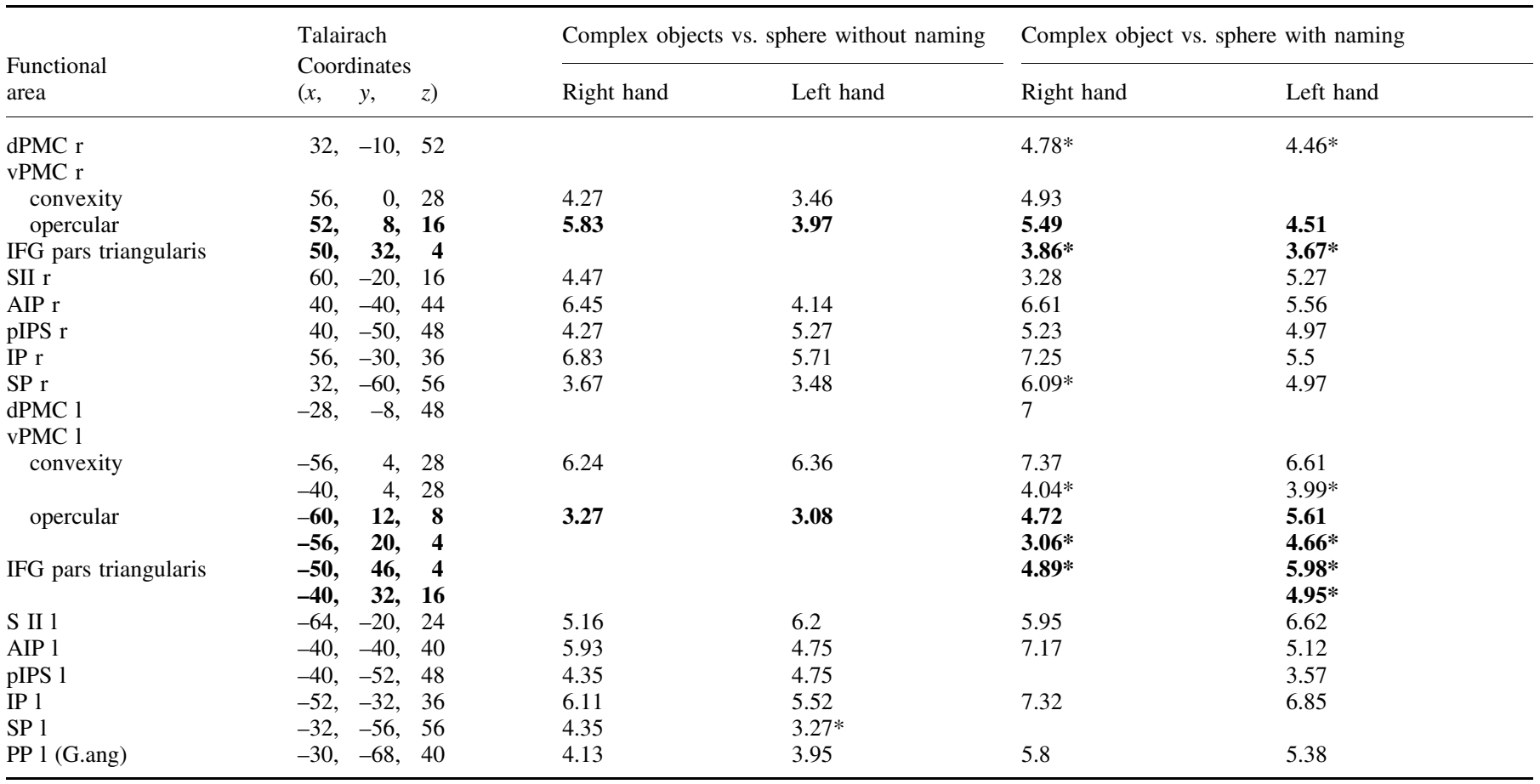

Z-scores are presented, with the premotor activation foci fitting into the probability maps of BAs 44 and 45 in bold. Abbreviations: 1, left; r, right; MI/SI, primary sensorimotor area; dPMC, dorsal premotor area; vPMC -ventral premotor area (convexity, on the precentral sulcus; opercular, on the pars opercularis, BA 44); IFG, inferior frontal gyrus (pars triangularis, BA 45); SII, secondary somatosensory area; AIP, anterior intraparietal area; pIPS, posterior intraparietal; IP, inferior parietal lobule; SP, superior parietal lobule; PP, posterior parietal area; G. ang., angular gyrus. *Activation significantly different between the two experimental conditions (with and without naming). 

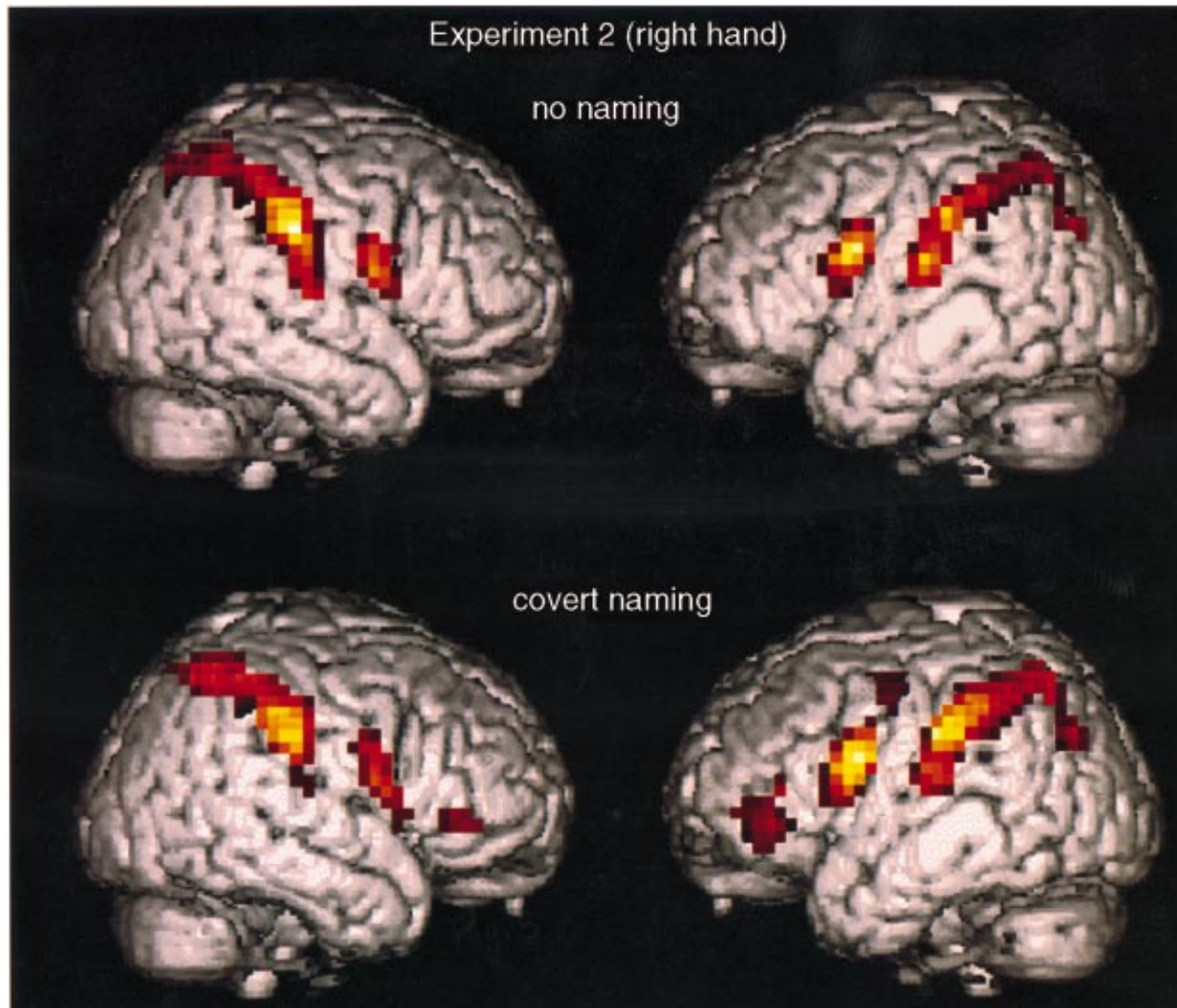

FIG. 2. Legend opposite.
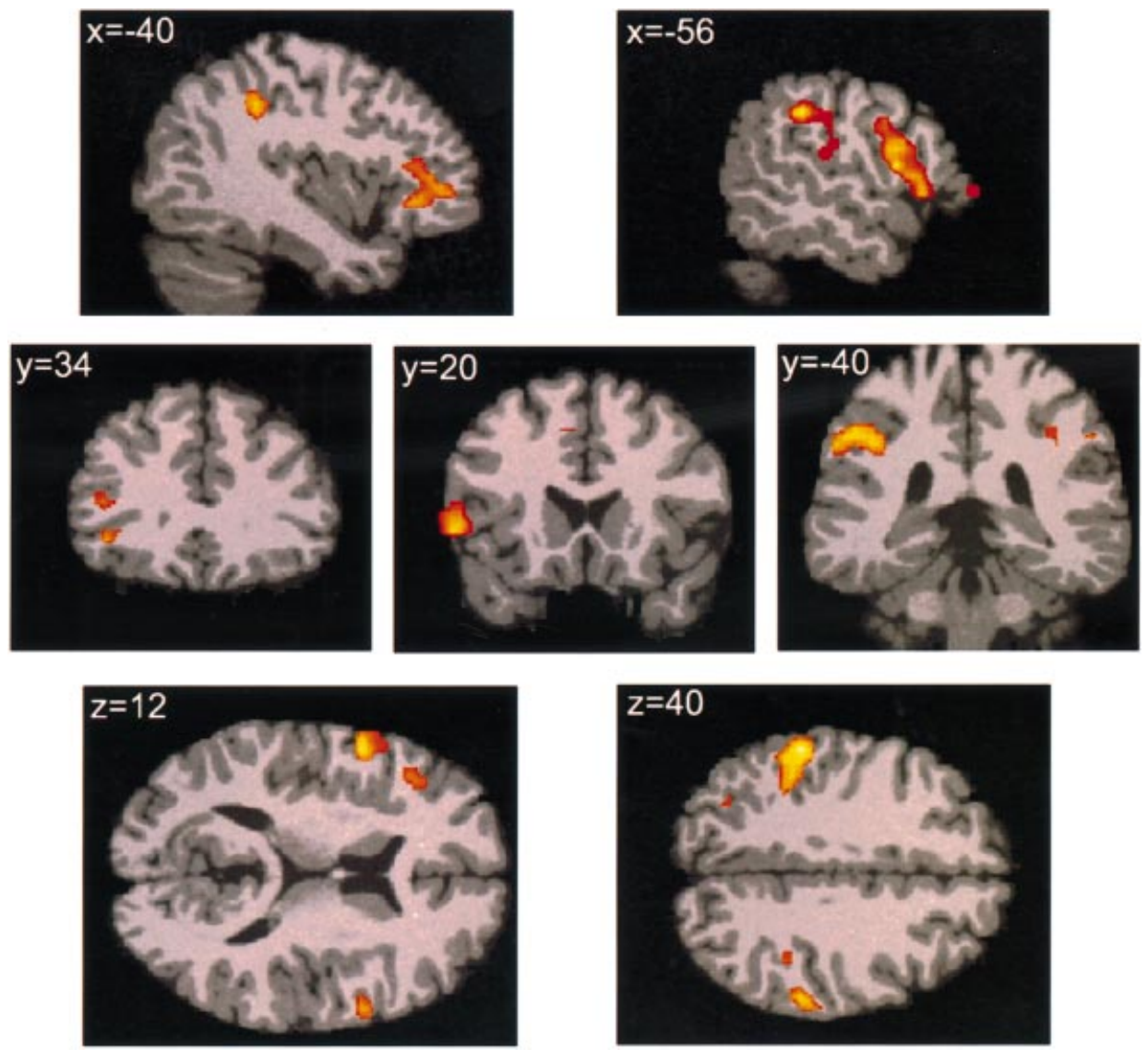

FIG. 3. Legend opposite. 
conditions of the experiment as projections on the lateral surface of a standard brain.

The comparison of complex manipulation without covert naming of the objects vs. simple manipulation confirmed the data of the first experiment. In addition, the data showed a further vPMC activation more ventrally located and a PP activity around the posterior angular gyrus.

The comparison of complex manipulation with covert naming vs. simple manipulation is shown in Table 2 and in Fig. 2. It is evident that additional activations in the more anterior opercular vPMC and, most importantly, in the triangular part of the inferior frontal gyrus are present in this condition. This area was active neither in experiment 1 nor in the condition of experiment 2 in which naming of the manipulated objects was not required (Table 2, Fig. 2).

The comparison of the coordinates of the activated foci located around the opercular and triangular parts of the inferior frontal gyrus with the coordinates of the probability maps of BAs 44 and 45 (Amunts et al., 1998, 1999) clearly demonstrated that the activation foci located in the pars triangularis related to covert naming of objects fitted entirely into BA 45 . The foci activated during complex object manipulation without naming and located in vPMC fitted into the borders of BA 44.

The more detailed anatomical locations of the ventral premotor and intraparietal foci from the second condition of experiment 2 are shown in the Fig. 3. The triangular focus is located beneath the anterior part of the inferior frontal sulcus (Fig. 3 upper panel, left; middle panel, left; lower panel, left). The opercular focus is located in the ventral frontal lobe anterior to the precentral sulcus (Fig. 3 upper panel, right; middle panel, middle; lower panel, left). The intraparietal focus is located on the lateral bank of the anterior intraparietal sulcus (Fig. 3 upper panel, left; middle panel, right; lower panel, right).

\section{Discussion}

The main finding of the present study is the demonstration that during manipulation of complex three-dimensional objects there are, in humans, selective activations of vPMC (BA 44), an area located in the anterior part of the lateral bank of the intraparietal sulcus (area AIP, BA 40) and of SII. An additional activation was present in the superior parietal lobule. If one compares these active sites with the areas that mediate grasping and manipulation in monkeys, it appears not only that, contrary to previous negative data (Grafton et al., 1996a; Matsumura et al., 1996; Rizzolatti et al., 1996; Faillenot et al., 1997), a circuit for hand-object interactions exists also in humans, but also that it is formed by the cortical areas that are usually considered to be the homologue of the monkey areas involved in the same function.

In the following sections we review the functional properties of the areas involved in hand-object interactions in humans and compare them with those of the putative homologous areas in the monkey. The influence of task design on the pattern of cerebral activation is also discussed.

\section{Ventral premotor cortex ( $V P M C)$}

In humans the ventral sector of the premotor cortex is formed by two areas: the ventral part of area 6a alpha and BA 44 (Vogt \& Vogt, 1919). The two areas share a common basic cytoarchitectonic structure, the main characteristics of which are the poverty (BA 44) or lack (BA 6) of granular cells (see Campbell, 1905; von Economo, 1929) and the presence of large pyramids in the third layer.

Classically, both ventral BA 6 and BA 44 were thought of as areas controlling oro-laryngeal movements, but with a different specialization and selectivity. The most lateral part of BA 6 was considered to be responsible of the motor control of buccal and laryngeal movements, regardless of the movement purpose, while, in contrast, BA 44 was considered to be the main speech motor area.

A series of recent studies showed that this view describes only partially the function of vPMC. These studies, based on brain imaging techniques, showed that a blood flow increase was present in vPMC during learning of finger movement sequences (Seitz \& Roland, 1992a), during mental imagery of grasping movements (Decety etal., 1994; Grafton etal., 1996b), during imaging of joystick movements (Stephan etal., 1995), during mental rotations necessary for hand recognition (Parsons etal., 1995), and during preparation of finger movements on the basis of a copied movement (Krams etal., 1998). The vPMC was also found to be of importance for motor tasks with high motor execution demands (Winstein etal., 1997). These data appear to suggest that, in addition to the control of oro-laryngeal movements, a representation of hand/arm movements is also contained in this area (Parsons et al., 1995; Preuss et al., 1996; Rizzolatti \& Arbib, 1998).

Until the present study, experiments in which the existence of a fronto-parietal circuit subserving hand-object interactions was overtly tested gave negative results (see Introduction). The most likely reason for this failure was the use of tasks based on discrete movements interrupted by long pauses. Such a paradigm (plus the simplicity of the required movements) is probably insufficient to activate the premotor cortex in a statistically significant way (see Grafton etal., 1996a).

In the present study we asked subjects to manipulate continuously complex objects and therefore continuously change finger configurations. Because the objects were placed into the subjects' hands and were obscured from the subjects' view, the subjects performed manipulatory finger movements induced by the macrostructure of the objects. We thought that such a task should be more effective in activating areas involved in finger control than those employed in previous experiments. The results confirmed this prediction. A marked activation was found in BA 44. This activation was bilateral, with a prevalence in the left hemisphere both when the task was executed with the right hand and when executed with the left hand (Table 1,2; Fig. 2).

FIG. 2. Projection of the activation foci from experiment 2 on the lateral surface of a standard brain from the Montreal Neurological Institute. Manipulation of complex objects vs. manipulation of a sphere. The right hand was used. Upper row, manipulation of objects that could not be named; lower row, manipulation of objects covertly named by the subjects. The red and yellow areas indicate pixels with Z-scores $>3.1$ (corrected $P<0.05$ ).

FIG. 3. Frontal and parietal activation foci projected on sections from a standard brain (Montreal Neurological Institute). Manipulation of complex objects with covert naming vs. manipulation of a sphere. Left side of the upper, middle and lower row: activation of the pars triangularis of the Broca's area; right side of the upper row, centre of the middle row and left side of the inferior row, activation of the opercular part of area 44; left side of the upper row, right side of the middle and lower rows, activation of the anterior part of the lateral bank of the intraparietal sulcus. The coordinates of the section planes are given in the upper left corner of each picture. 
These results fit well with the organization of vPMC in the monkey. As in humans, the vPMV in the monkey is constituted of two areas, F4 located caudally and F5 located rostrally (Matelli et al., 1985). Single-neuron recordings from F5 showed that in this area there are two large, partially overlapping, somatotopic fields, a hand field and a mouth field (Gentilucci et al., 1988). While the mouth field has not been much studied, there is evidence that hand-related neurons become active during goal-directed actions such a grasping, holding and manipulation (see Introduction and, for more details, Rizzolatti \& Fadiga, 1998).

It is interesting to note that a homology between BA 44 and area F5 was suggested in the 1940s by von Bonin \& Bailey (1947) on the basis of their cytoarchitectonic studies. (In their terminology, F5 is called FCBm.) This view was recently fully supported by Petrides \& Pandya (1994; see also Galaburda \& Pandya, 1982; Preuss etal., 1996).

A possible weakness of this homology (see Passingham, 1993) is the richness of the oro-laryngeal representation, including that of speech control, in humans and, on the contrary, the presence of an important finger-movement representation in monkeys. The present data indicate that a hand/finger representation is indeed also present in human BA 44. Obviously, the relative cortical space for the two representations is not the same. However, the development of the cortex devoted to oro-laryngeal representations specifically in BA 44 is probably not a mere coincidence, but is due to the close evolutionary relation between action and speech (see Rizzolatti \& Arbib, 1998).

In the condition in which we presented meaningful stimuli and asked subjects to name them, the results showed additional frontal lobe activations (experiment 2). They were located in the opercular part of the BA 44 and in BA 45 . Note that the activation of BA 45 was never present when the subjects merely manipulated objects. These activation could be due either to object naming (see Frith et al., 1991; Warburton etal., 1996; Paulesu etal., 1997) or to their recognition (Perani et al., 1995; Grafton et al., 1997). Rostral BA 45 was found to be involved in both these functions.

\section{Intraparietal sulcus}

Posterior parietal lobule lesions involving the superior parietal lobe and the adjacent areas of the intraparietal sulcus are known to produce reaching deficits (Balint, 1909; see also De Renzi, 1982; Perenin \& Vighetto, 1988). Although less frequently reported, another important impairment in sensorimotor and visuomotor behaviour following posterior parietal damage is an inadequate hand and finger shaping (Jeannerod, 1986; Pause etal., 1989; Binkovski etal., 1992).

Until recently very little was known about the location of the finger/hand movement representation in human parietal cortex. Recently, evidence has been provided that grasping finger movements are localized in the cortex, located in the anterior part of the lateral bank of the intraparietal sulcus. Binkofski et al. (1998a) reported that, after a lesion centred in this region, patients show selective deficits in the co-ordination of finger movements required for object grasping, their reaching movements being only mildly disturbed. Moreover, the same focus was activated as evident from fMRI, when healthy subjects performed prehension movements (Binkofski etal., 1998a).

The present study confirms this localization (Table 1,2). During complex object manipulation an activation was found in the cortex located in the intraparietal sulcus. The active area lay in the rostral part of the sulcus in correspondence with its lateral bank (BA 40).
An activation within the right intraparietal sulcus was found by Faillenot etal. (1997) using a visual object matching task. This activation was interpreted as related to visual recognition of invariant features of objects. No activation of the intraparietal sulcus was found during grasping. The activation of the inferior part of the left postcentral gyrus (BA 2/40) found in this condition was probably due to proprioceptive afferences related to finger movements. An activation similar to that observed by Faillenot et al. (1997) was recently reported by Taira et al. (1998) in a visual axis discrimination task.

An activation of BA 40 was found also in mental imagery of grasping as well as trajectory movements (Grafton et al., 1996b; Seitz etal., 1997). These activation sites, however, were located more posteriorly than that described in our study. This difference might be explained by postulating that, as in SMA proper (Tyszka et al., 1994; Roth et al., 1995; Grafton etal., 1996b), in the intraparietal sulcus the region for imagined movements is close to but distinct from that for actual movements. It may also be, however, that the observed different locations between true and imagined movements are not due to a real functional differentiation inside the sulcus, but to intersubject variability or to methodological factors. Finally, it is important to note that the 'grasping' studies that failed to reveal a premotor activation during grasping movements also failed to find an activation of AIP. This finding further supports the view that the task used in those experiments was inadequate for exciting the human circuit responsible for hand-object interactions.

In conclusion, it appears that in humans as in monkeys there is a parieto-frontal circuit for hand-object interactions. The parietal node of this circuit is area AIP in the monkey and the intraparietal area activated in the present study in humans. It is important to stress that AIP neurons do not discharge only during object presentation and visually-guided hand shaping, but also during object holding and manipulation (Sakata et al., 1992, 1995; Jeannerod etal., 1995). Furthermore the AIP neuron types defined as 'motor dominant' and 'visual-and-motor' discharge during hand-related actions performed in the dark. Thus, the activation of human intraparietal sulcus during movements executed without visual guidance is in full accord with the nerophysiological data on monkey area AIP.

\section{Area SII}

The second somatosensory area (SII) in primates, including humans, lies mostly in the upper bank of the Sylvian fissure, immediately posterior to the central sulcus (Penfield \& Jasper, 1954; Woolsey, 1958; Whitsel etal., 1969; Lüders etal., 1985; Kaas \& Pons, 1988; Burton etal., 1993). In human imaging studies SII has been shown to be activated by strong somatosensory stimuli such as vibration and somatic pain (Seitz \& Roland, 1992b; Talbot etal., 1991; Binkofski etal., 1998b). Recent studies in the monkey showed that the SII of classical authors is formed by two separate areas both sensitive to tactile stimuli: the parietal ventral area (PV) located rostrally and SII caudally (Krubitzer etal., 1995). Furthermore around the PV/SII complex there are other cortical fields that also respond to somatosensory stimuli (Robinson \& Burton, 1980; Krubitzer et al., 1995). In the present study the term SII will be used in a broader sense indicating both the small, strictly defined SII and the adjacent somatosensory fields.

Anatomical studies in monkey showed that SII has connections with vPMC including F5, with area 7b, and with different sectors of the insula (Pandya \& Kuypers, 1969; Mufson et al., 1981; Friedman etal., 1986; Matelli etal., 1986). Thus, SII conveys somatosensory 
information to motor areas on one side and to the limbic system on the other side.

Lesion studies showed that following ablation of SII, monkeys are severely impaired in tactile learning and retention of shapes (Ridley \& Ettlinger, 1976, 1978; Murray \& Mishkin, 1984), while their basic tactile sensory capacities remain intact (Ridley \& Ettlinger, 1976, 1978; Garcha \& Ettlinger, 1978). On the basis of these findings and other considerations, Mishkin (1979) proposed that SII plays a central role in tactile-affective associations, similar to that attributed to infero-temporal cortex in vision.

Both stimulation and lesion studies of SII are rare in humans. Cortical stimulation in awake patients typically causes simple sensory sensations (Lüders etal., 1985). Focal lesions of the parietal operculum that included SII produce tactile agnosia without loss of simple tactile sensation or motor control (Caselli, 1991, 1993). The deficit can include the inability to classify objects on the basis of their size or shape.

In the present study an activation of SII (and adjacent areas) was observed in all tasks of our experiment (Table 1,2). This activation was particularly strong in the condition in which complex object manipulation was compared to sphere manipulation. Because no concomitant, significant activation was found in SI in this last condition, what might appear at first glance the simplest explanation of this finding is rather unlikely: that the increase of activation in SII during the task was exclusively due to the different amount of somatosensory stimulation.

Once this explanation is discarded, what can be the reason for the increase of SII activity during complex object manipulation? If one considers the duality of efferent connections of SII, linking SII on one side with the insula and on the other with vPMC, two possibilities appear to be particularly plausible. The first is that the activation of SII is related to object discrimination. Although no overt object discrimination was required in our first experiment, it might be that this process was automatically triggered by the task. Against this interpretation are, however, the findings of Grafton et al. (1996a) and Faillenot et al. (1997) who reported an activation of SII in a grasping vs. pointing task in which no tactile object discrimination was present. An alternative possibility is that the somatosensory information conveyed by SII to vPMC was used to control and direct finger movement during object exploration in such a way as to adapt the finger grip to the object's intrinsic features in absence of visual control. This interpretation is consistent with the notion that F5 needs a continuous flow of tactile information. This information is needed both for F5 'grasping' neurons as a signal that the target has been reached and for F5 'holding' neurons which discharge when a contact between finger and object is established. Our view is that SII provides this indispensable tactile input to vPMC.

\section{Superior parietal lobule}

In all primates, including the prosimians, the intraparietal sulcus divides the posterior part of the parietal lobe into two sectors, the superior parietal lobule and the inferior parietal lobule. According to Brodmann (1909) each parietal lobule is formed by two cytoarchitectonic areas: (i) the superior parietal lobule, formed by BA 5 and 7 , and (ii) the inferior parietal lobule, formed by BA 39 and 40 . In his map of monkey brain Brodmann considered the monkey superior parietal lobule to be constituted of an area homologous to human area 5 and the inferior parietal lobule of an area homologous to human area 7. This implies that, in evolution, the non-human area 7 had 'jumped' from its original location below the intraparietal sulcus to a location above it. This very surprising view was not confirmed by von
Bonin \& Bailey, (1947). Following von Economo (1929), they found in both humans and monkey a main cytoarchitectonic area in the superior parietal lobule called area $\mathrm{PE}$, and two areas in the inferior parietal lobule, areas PF and PG.

Because of the popularity of Brodmann's human cortical map, the homology proposed by Brodmann has been the source of considerable confusion and the properties of monkey area 7 were often attributed to human superior parietal lobule. In the following discussion we will use exclusively the homology of von Bonin and Bailey: only the data derived from the study of the monkey superior parietal lobule will be used in discussing the superior parietal lobule in humans.

In monkeys the superior parietal lobule is essentially related to the elaboration of proprioceptive information. Neurons from area PE, the area forming most of the superior parietal lobule cortical convexity, are active with passive joint rotation and deep tissue pressure as well as during active arm movements (Sakata etal., 1973; Mountcastle et al., 1975; Kalaska etal., 1983; Lacquaniti et al., 1995). Some of them combine proprioceptive information from different joints, possibly playing a role in a more global representation of body parts (Mountcastle etal., 1975), others put together tactile and joint information (Sakata etal., 1973). Recent evidence has shown that, while PE is exclusively related to somatosensory modalities, the posterior sectors of the superior parietal lobule (e.g. area V6 A, Galetti et al., 1996) have in addition visual functions (see references in Rizzolatti et al., 1997).

Is there a hand/finger representation in human superior parietal lobule? There are not many data on this point, most of the studies on the superior parietal lobule concerning global arm movements (e.g. Roland etal., 1980; Deiber etal., 1991; Grafton et al., 1992) rather than pure hand/finger movements. Evidence, however, in favour of such a representation has been reported by Seitz et al. (1991) who asked subjects to discriminate among a series of cuboids differing only in their length. The results showed an increase of cerebral blood flow in the primary sensory and motor areas, in premotor cortex, in the supplementary motor area and, most importantly for the present discussion, in the superior parietal lobule.

The presence of a hand/finger representation in the superior parietal lobule was demonstrated also by a clinical study in which patients with anterior parietal lesions were compared with patients with posterior parietal lesions mostly involving the superior parietal lobule (Pause et al., 1989). When the damage was anterior, the simple aspects of somaesthesis were strongly disturbed, while somaesthesia was preserved when the damage was located in the posterior parietal cortex. In the latter condition hemiparesis was only mild or absent, whereas exploratory and manipulative finger movements were severely impaired. Remarkably, the exploratory finger movements could be produced by imitation. Furthermore, hand shaping and target acquisition in visuomotor tasks were less disturbed than manipulative behaviour.

The presence of a hand/finger representation in the superior parietal lobule was confirmed by the present findings (Table 1,2). They also showed an intense activation of the superior parietal lobule during hand manipulation of three-dimensional objects.

\section{Representations of manipulation in parietal cortex}

The presence of two hand/finger representations, one in SII and in the other in the superior parietal lobule, both related to somatosensory modalities, raises the question of their relative contribution to manipulative behaviour. A clue for answering this question can be obtained (in addition to the data reported above) by the neuron properties of the two areas as reported in monkey studies. These 
studies show that the large majority of SII neurons are responsive to tactile and not to joint stimulation (Robinson \& Burton, 1980) while, in contrast, area PE is mostly related to proprioception, only a small number of neurons responding to tactile stimulation (see above). Our suggestion is therefore the following: both PE and SII are involved in controlling exploratory manipulation. Their role, however, is different. SII and the adjacent areas (SII stream) describe the objects in terms of their intrinsic (physical) properties. In contrast, PE and the adjacent areas (superior parietal stream) describe the objects in terms of hand postures necessary to interact with them. The functional role of SII is therefore to capture information from the external world, whereas that of PE is to describe the same objects from an internal (kinaesthetic) point of view. The AIP seems to play an intermediate role, as it processes information required for initiating hand-object interaction. Finally, although both streams cooperate in object manipulation, the greater sensitivity of the tactile modality with respect to the kinaesthetic modality (Roland, 1987), the anatomical connections of SII with the limbic system (see Mishkin, 1979), and the ablation experiments reviewed above, all indicate that the SII stream plays a major role in tactile object identification.

\section{Acknowledgements}

Comments from K. Amunts concerning anatomical locations of premotor activations are gratefully acknowledged. This study was supported by the Deutsche Forschungsgemeinschaft (SFB 194) and by a BioMed grant to G.R.

\section{Abbrevations}

AIP, anterior intraparietal area; BA, Brodmann's area; dPMC, dorsal premotor cortex; fMRI, functional magnetic resonance imaging; FOV, field of view; mCing, cingulate motor cortex; MI, primary motor cortex; MR, magnetic resonance; PET, positron emission tomography; PP, posterior part of the superior parietal lobule; SI, primary somatosensory area; SII, secondary somatosensory area; SMA, supplementary motor area; SP, superior parietal lobule; TE, signal (echo)-gathering time; TR, sequence repetition time; $\mathrm{vPMC}$, ventral premotor cortex.

\section{References}

Amunts, K., Klingberg, T., Binkofski, F., Schormann, T., Seitz, R.J., Roland, P.E. \& Zilles, K. (1998) Cytoarchitectonic definition of Broca's region and its role in functions different from speech. Neuroimage, 7, 8.

Amunts, K., Schleicher, A., Bürgel, U., Mohlberg, H., Uylings, H.B.M. \& Zilles, K. (1999) Broca's region re-visited: Cytoarchitecture and intersubject variability. J. Comp. Neurol., in press.

Balint, A. (1909). Seelenlähmung des Schauens, optische Ataxie, räumliche Störung der Aufmerksamkeit. Monatsschr. Psychiatr. Neurol., 25, 51-81.

Binkofski, F., Dohle, C., Posse, S., Stephan, K.M., Hefter, H., Seitz, R.J. \& Freund, H.-J. (1998a) Human anterior intraparietal area subserves prehension. A combined lesion and functional MRI activation study. Neurology, 50, 1253-1259.

Binkofski, F., Kunesch, E. \& Freund, H.-J. (1992) Disturbance of spatiotemporal patterns of exploratory finger movements in patients with parietal lesions. Soc. Neurosci. Abstr., 18, 281.286.

Binkofski, F., Schnitzler, A., Enck, P., Frieling, T., Posse, S., Seitz, R.J. \& Freund. H.-J. (1998b) Somatic and limbic activations in esophageal distention. Ann. Neurol., 44, 811-815.

von Bonin, G. \&. Bailey, P. (1947) The Neocortex of Macaca Mulatta. University of Illinois Press, Urbana.

Brodmann, K. (1909) Vergleichende Lokalisationslehre der Großhirnrinde. Barth, Leipzig.

Burton, H., Videen, T.O. \& Reichle, M.E. (1993) Tactile-vibration-activated foci in insular and parietal opercular cortex with positron emission tomography: mapping the second somatosensory area in humans. Somatosens. Motor Res., 10, 297-308.

Campbell, F. (1905) Histological studies on localization of cerebral function. Cambridge University Press, Cambridge.
Caselli, R.J. (1991) Rediscovering tactile agnosia. Mayo Clin. Proc., 66, 19 42.

Caselli, R.J. (1993) Ventrolateral and dorsomedial somatosensory association cortex damage produces distinct somesthetic syndromes in humans. Neurology, 43, 762-771.

Colebatch, J.G., Deiber, M.P., Passingham, R.E., Friston, K.J. \& Frackowiak. R.S.J. (1991) Regional cerebral blood flow during voluntary arm and hand movements in human subjects. J. Neurophysiol., 65, 1392-1401.

De Renzi, E. (1982) Disorders of space exploration and cognition. Wiley, New York.

Decety, J., Perani, D., Jeannerod, M., Bettinardi, V., Tadary, B., Woods, R., Mazziotta, J.C. \& Fazio, F. (1994) Mapping motor representations with positron emission tomography. Nature, 371, 600-602.

Deiber, M.P., Passingham, R.E., Colebatch, J.G., Friston, K.J., Nixon, P.D. \& Frackowiak, R.S.J. (1991) Cortical areas and the selection of movement: a study with positron emission tomography. Exp. Brain Res., 84, 392-402.

von Economo, C. (1929) The Cytoarchitecture of the Human Cerebral Cortex. Oxford University Press, London.

Faillenot, I., Toni, I., Decety, J., Gregoire, M.C. \& Jeannerod, M. (1997) Visual pathways for object-oriented action and object recognition: functional anatomy with PET. Cerebral Cortex, 7, 77-85.

Fink, G.R., Frackowiak, R.S.J., Pietrzyk, U. \& Passingham, R.E. (1997) Multiple nonprimary motor areas in the human cortex. J. Neurophysiol., 77, 2164-2174.

Friedman, D.P., Murray, E.A., O’Neil, J.B. \& Mishkin, M. (1986) Cortical connections of the somatosensory fields of the lateral sulcus of macaques: evidence for a corticolimbic pathway for touch. J. Comp. Neurol., 252, 323347.

Friston, K.J. (1995a) Commentary and opinion: II. Statisical parametric mapping: ontology and current issues. J. Cereb. Blood Flow Metab., 15, 361-370.

Friston, K.J., Ashburner, J., Poline, J.B., Frith, C.D., Heather, J.D. \& Frackowiak, R.S.J. (1997) Spatial realignment and normalization of images. Hum. Brain Mapp., 2, 165-189.

Friston, K.J., Holmes, A.P., Poline, J.B., Grasby, P.J., Wiliams, S.C.R., Frackowiak, R.S.J. \& Turner, R. (1995b) Analysis of fMRI time-series revisited. Neuroimage, 2, 5-53.

Friston, K.J., Jezzard, P. \& Turner, R. (1994a) Analysis of functional MRI time-series. Hum. Brain Mapp., 1, 153-171.

Friston, K.J., Worsley, K.J., Frackowiak, R.S.J., Maziotta, J.C. \& Evans, A.C. (1994b) Assessing the significance of focal activation using their spatial extent. Hum. Brain Mapp., 1, 210-220.

Frith, C.D., Friston, K.J., Liddle, P.F. \& Frackowiak, R.S.J. (1991) A PET study of word finding. Neuropsychologia, 29, 1137-1148.

Galaburda, A.M. \& Pandya, D.N. (1982) Role of architectonics and connections in the study of primate brain evolution. In Armstrong, E. \& Falk, D. (eds), Primate Brain Evolution: Methods and Concepts. Plenum Press, New York, pp. 203-216.

Galetti, C., Fattori, P., Battaglini, P.P., Shipp, S. \& Zeki, S. (1996) Functional demarcation of a border between area V6 and V6A in the superior parietal gyrus of the macaque monkey. Eur. J. Neurosci., 8, 30-52.

Garcha, H.S. \& Ettlinger, G. (1978) The effects of unilateral or bilateral removals of the second somatosensory cortex (area SII): a profound tactile disorder in monkeys. Cortex, 14, 319-326.

Gentilucci, M., Fogassi, L., Luppino, G., Matelli, M., Camarda, R. \& Rizzolatti, G. (1988) Functional organization of inferior area 6 in the macaque monkey: I. Somatotopy and the control of proximal movements. Exp. Brain Res., 71, 475-490.

Godschalk, M., Lemon, R.N., Kuypers, H.G.J.M. \& Ronday, H.K. (1984) Cortical afferents and efferents of monkey postarcuate area: an anatomical and electrophysiological study. Exp. Brain Res., 56, 410-424.

Grafton, S.T., Arbib, M.A., Fadiga, L. \& Rizzolatti, G. (1996b) Localization of grasp representation in humans by position emission tomography: 2 . Observation compared with imagination. Exp. Brain Res., 112, 103-111.

Grafton, S.T., Fadiga, L., Arbib, M.A. \& Rizzolatti, G. (1997) Premotor cortex activation during observation and naming of familiar tools. Neuroimage, $\mathbf{6}$, 231-236.

Grafton, S.T., Fagg, A.H., Woods, R.P. \& Arbib, M.A. (1996a) Functional anatomy of pointing and grasping in humans. Cereb. Cortex, 6, 226-237.

Grafton, S.T., Mazziotta, J.C., Woods, R.P. \& Phelps, M.E. (1992) Human functional anatomy of visually guided finger movements. Brain, 115, 565587.

Hepp-Raymond, M.C., Husler, E.J., Maier, M.A. \& Qi, H.X. (1994) Forcerelated neuronal activity in two regions of the primate ventral premotor cortex. Can. J. Physiol. Pharmacol., 72, 571-579. 
Jeannerod, M. (1986) The formation of finger grip during prehension. A cortically mediated visuomotor pattern. Behav. Brain Res., 19, 99-116.

Jeannerod, M., Arbib, M.A., Rizzolatti, G. \& Sakata, H. (1995) Grasping objects: the cortical mechanism of visuomotor transformation. Trends Neurosci., 18, 314-320.

Kaas, J.H. \& Pons, T.P. (1988) The somatosensory system of primates. In Steklis, H.P. (ed.), Comparative Primate Biology. Liss, New York.

Kalaska, J.F., Caminiti, R. \& Georgopoulos, A.P. (1983) Cortical mechanisms related to the direction of two-dimensional arm movements: relations in parietal area 5 and comparison with motor cortex. Exp. Brain Res., 51, 247260.

Krams, M., Rushworth, M.F.S., Deiber, M.P., Frackowiak, R.S.J. \& Passingham, R.E. (1998) The preparation, execution and suppression of copied movements in the human brain. Exp. Brain Res., 120, 386-398.

Krubitzer, L., Clarey, J., Tweedale, R., Elston, G. \& Calford, M. (1995) A redefinition of somatosensory areas in the lateral sulcus of Macaque monkeys. J. Neurosci., 15, 3821-3839.

Kunesch, E., Binkofski, F. \& Freund, H.-J. (1989) Invariant temporal characteristics of manipulative hand movements. Exp. Brain Res., 78, 539-546.

Kurata, K. \& Tanji, J. (1986) Premotor cortex neurons in macaques: activity before distal and proximal forelimb movements. J. Neurosci., 6, 403-411.

Lacquaniti, F., Guigon, E., Bianchi, L., Ferraini, S. \& Caminiti, R. (1995) Representing spatial information for limb movement: role of area 5 in the monkey. Cereb. Cortex, 5, 391-409.

Lüders, H., Lesser, R.P., Dinner, D.S., Hahn, J.F., Salanga, V. \& Morris, H.H. (1985) The second sensory area in humans: evoked potential and electrical stimulation studies. Ann. Neurol., 17, 177-184.

Luppino, G., Murata, A., Govoni, P. \& Matelli, M. (1999) Independent parieto-frontal circuits linking rostral intraparietal cortex (areas AIP and VIP) and the ventral premotor cortex (areas F4 and F5). J. Comp. Neurol., (in press).

Matelli, M., Camarda, R., Glickstein, M. \& Rizzolatti, G. (1986) Afferent and efferent projections of the inferior area 6 in the macaque monkey. J. Comp. Neurol., 251, 281-298.

Matelli, M., Luppino, G. \& Rizzolatti, G. (1985) Patterns of cytochrome oxidase activity in the frontal agranular cortex of macaque monkey. Behav. Brain Res., 18, 125-137.

Matsumura, M., Kawashima, R., Naito, E., Satoh, K., Takahashi, T., Yanagisawa, T. \& Fukuda, H. (1996) Changes in rCBF during grasping in humans examined by PET. Neuroreport, 7, 749-752.

Matsumura, M. \& Kubota, K. (1979) Cortical projections of hand-arm motor area from postarcuate area in macaque monkey: a histological study of retrograde transport of horseradish peroxidase. Neurosci. Lett., 11, 241-246.

Mishkin, M. (1979) Analogous neural models for tactual and visual learning. Neuropsychologia, 17, 139-150.

Mountcastle, V.B., Lynch, J.C.G.A., Sakata, H. \& Acuna, C. (1975) Posterior parietal association cortex of the monkey: command functions for operations within extrapersonal space. J. Neurophysiol., 38, 871-908.

Muakkassa, K.F. \& Strick, P.L. (1979) Frontal lobe inputs to primate motor cortex: evidence for four somatotopically organized 'premotor' areas. Brain Res., 177, 176-182.

Mufson, E.J., Mesulam, M.M. \& Pandya, D.N. (1981) Insular interconnections with the amygdala in the rhesus monkey. Neuroscience, 6, 1231-1248.

Murata, A., Fadiga, L., Fogassi, L., Gallese, V., Raos, V. \& Rizzolatti, G. (1997) Object representation in the ventral premotor cortex (area F5) of the monkey. J. Neurophysiol., 78, 2226-2230.

Murray, E.A. \& Mishkin, M. (1984) Relative contributions of SII and area 5 to tactile discrimination in monkeys. Behav. Brain Res., 11, 67-83.

Oldfield, R.C. (1971) The assessment and analysis of handedness: the Edinburgh inventory. Neuropsychologia, 87, 256-259.

Pandya, D. \& Kuypers, H.G.J.M. (1969) Cortico-cortical connections in the rhesus monkey. Brain Res., 13, 13-36.

Parsons, L.M., Fox, P.T., Hunter Downs, J., Glass, T., Hirsch, T.B., Martin, C.C., Jerabek, P.A. \& Lancaster, J.L. (1995) Use of implicit motor imagery for visual shape discrimination as revealed by PET. Nature, 375, 54-58.

Passingham, R. (1993) The Frontal Lobes and Voluntary Actions. Oxford University Press, Oxford.

Paulesu, E., Goldacre, B., Scifo, P., Cappa, S.F., Gilardi, M.C., Castiglioni, L., Perani, D. \& Fazio, F. (1997) Functional heterogeneity of left frontal cortex as revealed by fMRI. Neuroreport, 8, 2011-2016.

Pause, M., Kunesch, E., Binkofski, F. \& Freund, H.-J. (1989) Sensorimotor disturbances in patients with lesions of the parietal cortex. Brain, 112, 1599-1625.
Penfield, W. \& Jasper, H. (1954) Epilepsy and the Functional Anatomy of the Human Brain. Little Brown, Boston.

Perani, D., Cappa, S.F., Bettinardi, V., Bressi, S., Gorno-Tempini, M., Mataresse, M. \& Fazio, F. (1995) Different neural systems for recognition of animals and manmade tools. Neuroreport, 6, 1637-1641.

Perenin, M.T. \& Vighetto, A. (1988) Optic ataxia: specific disruption in visuomotor mechanisms. Brain, 111, 643-674.

Petrides, M. \& Pandya, D.N. (1994) Comparative architectonic analysis of the human and macaque frontal cortex. In Grafman, J. \& Boller, F. (eds), Handbook of Neuropsychology. Elsevier, Amsterdam.

Poline, J.B., Worsley, K.J., Holmes, A.P., Frackowiak, R.S.J. \& Friston, K.J. (1995) Estimating smoothness in statistical parametric maps: variability of P-values. J. Comput. Assist. Tomogr., 19, 788-796.

Porter, R. \& Lemon, R. (1993) Corticospinal Function and Voluntary Movement. Clarendon Press, Oxford.

Preuss, T.M., Stepniewska, I. \& Kaas, J.H. (1996) Movement representation in the dorsal and ventral premotor areas of owl monkeys: a microstimulation study. J. Comp. Neurol., 371, 649-675.

Ridley, R.M. \& Ettlinger, G. (1976) Impaired tactile learning and retention after removals of the second somatic sensory projection cortex (SII) in the monkey. Brain Res., 109, 656-660.

Ridley, R.M. \& Ettlinger, G. (1978) Further evidence of impaired tactile learning after removals of the second somatic sensory projection cortex (SII) in the monkey. Exp. Brain Res., 32, 475-480.

Rizzolatti, G. \& Arbib, M.A. (1998) Language within our grasp. Trends Neurosci., 21, 188-194.

Rizzolatti, G., Camarda, R., Fogassi, L., Gentilucci, M., Luppino, G. \& Matelli, M. (1988) Functional organization of inferior area 6 in the macaque monkey. II: area F5 and the control of distal movements. Exp Brain Res., 71, 491-507.

Rizzolatti, G. \& Fadiga, L. (1998) Grasping objects and grasping action meanings: the dual role of monkey rostroventral premotor cortex (area F5). In Sensory Guidance of Movements (Novartis Foundation Symposium 218). Wiley, Chichester, pp. 81-103.

Rizzolatti, G., Fadiga, L., Matelli, M., Bettinardi, V., Paulesu, E., Perani, D. \& Fazio, F. (1996) Localization of grasp representation in humans by PET: I. Observation versus execution. Exp. Brain Res., 111, 246-252.

Rizzolatti, G., Fogassi, L. \& Gallese, V. (1997) Parietal cortex: from sight to action. Curr. Opin. Neurobiol., 7, 562-567.

Rizzolatti, G., Scandolara, C., Matelli, M. \& Gentilucci, M. (1981) Afferent properties of peri-arcuate neurons in macaque monkeys. II. Somatosensory responses. Behav. Brain Res., 2, 147-163.

Robinson, C.J. \& Burton, H. (1980) Somatotopographic organization in the second somatosensory area of M. fascicularis. J. Comp. Neurol., 192, 43 67.

Roland, P.E. (1987) Somatosensory detection of microgeometry, macrogeometry and kinesthesia after localized lesions of the cerebral hemiparesis in man. Brain Res. Rev., 12, 43-94.

Roland, P.E., Steinhof, E., Larsen, N.A. \& Larsen, B. (1980) Differential areas in man in organization of voluntary movement in extrapersonal space. $J$. Neurophysiol., 43, 137-150.

Roland, P.E., Meyer, E., Yamamoto, Y.L. \& Thompson, C.J. (1982) Regional cerebral blood flow changes in cortex and basal ganglia during voluntary movements in normal human volunteers. J. Neurophysiol., 48, 467-480.

Roland, P.E. \& Zilles, K. (1996) Functions and structures of the motor areas in humans. Curr. Opin. Neurobiol., 6, 773-781.

Roth, M., Decety, J., Raybaudi, M., Massarelli, R., Delon-Martin, C., Segebarth, C., Morand, S., Gemignani, A., Decorps, M. \& Jeannerod, M. (1995) Possible involvement of primary motor cortex in mentally simulated movements: a functional magnetic imaging study. Neuroreport, 7, 12801284.

Sakata, H., Taira, M. \& Murata, A. (1992) Hand-movements related neurons of the posterior parietal cortex of the monkey: their role in visual guidance of hand movements. In Caminiti, R., Johnson, P.B. \& Burnod, Y. (eds), Control of Arm Movements in Space: Neurophysiological and Computational Approaches. Springer, Berlin.

Sakata, H., Taira, M., Murata, A. \& Mine, S. (1995) Neural mechanisms of visual guidance of hand action in the parietal cortex of the monkey. Cereb. Cortex, 5, 429-438.

Sakata, H., Takaoka, Y., Kawasaki, A. \& Shibutani, H. (1973) Somatosensory properties of neurons in the superior parietal cortex (area 5) of the rhesus monkey. Brain Res., 64, 85-102.

Schlaug, G., Knorr, U. \& Seitz, R.J. (1994) Inter-subject variability of cerebral activations in acquiring a motor skill: a study with PET. Exp. Brain Res., 98, $523-534$. 
Seitz, R.J., Canavan, A.G.M., Yaguez, L., Herzog, H., Tellmann, L., Knorr, U., Huang, Y. \& Homberg, V. (1997) Representations of graphomotor trajectories in human parietal cortex: evidence for controlled processing and automatic performance. Eur. J. Neurosci., 9, 378-389.

Seitz, R.J. \& Roland, P.E. (1992a) Learning of sequential finger movements in man: a combined kinematic and positron emission tomography (PET) study. Eur. J. Neurosci., 4, 154-165.

Seitz, R.J. \& Roland, P.E. (1992b) Vibratory stimulation increases and decreases the regional cerebral blood flow and oxidative metabolism: a postitron emission tomography (PET) study. Acta Neurol. Scand., 86, 60-67.

Seitz, R.J., Roland, P.E., Bohm, C., Greitz, T. \& Stone-Elander, S. (1991) Somatosensory discrimination of shape: tactile exploration and cerebral activation. Eur. J. Neurosci., 3, 481-492.

Stephan, K.M., Fink, G., Passingham, R.E., Silberzweig, D., CeballosBaumann, A.O., Frith, C.D. \& Frackowiak, R.S.J. (1995) Functional anatomy of the mental representation of upper extremity movements in health subjects. J. Neurophysiol., 73, 373-386.

Taira, M., Kawashima, R., Inoue, K. \& Fukuda, H. (1998) A PET study of axis orientation discrimination. Neuroreport, 9, 283-288.

Taira, M., Mine, S., Georgopoulos, A.P., Murata, A. \& Sakata, H. (1990) Parietal cortex neurons of the monkeys related to the visual guidance of hand movement. Exp. Brain Res., 83, 29-36.

Talairach, J. \& Tournoux, P. (1988) Co-Planar Stereotactic Atlas of the Human Brain. Thieme Medical Publishers, Stuttgart, New York.
Talbot, J.D., Marret, S., Evans, A.C., Meyer, E., Bushnell, M.C. \& Ducan, G.H. (1991) Multiple representations of pain in human cerebral cortex. Science, 251, 1355-1358.

Tyszka, J.M., Grafton, S.T., Chew, W., Woods, R.P. \& Colletti, P.M. (1994) Parcellation of mesial frontal motor areas during ideation and movement using functional magnetic resonance imaging at 1.5 Tesla. Ann. Neurol., 35 , 746-749.

Vogt, O. \& Vogt, C. (1919) Ergebnisse unserer Hirnforschung. J. Psychol. Neurol., 25, 277-462.

Warburton, E., Wise, R.J.S., Price, C.J., Weiler, C., Hadar, U., Ramsay, S. \& Frackowiak, R.S.J. (1996) Noun and verb retrieval by normal subjects. Brain, 119, 59-79.

Whitsel, B.L., Petrucelli, L.M. \& Werner, G. (1969) Symmetry and connectivity in the map of the body surface in somatosensory area II of primates. J. Neurophysiol., 32, 170-183.

Winstein, C.J., Grafton, S.T. \& Pohl, P.S. (1997) Motor task difficulty and brain activity: investigation of goal directed reciprocal aiming using positron emission tomography. J. Neurophysiol., 77, 1581-1594.

Woolsey, C.N. (1958) Organization of somatic sensory and motor areas of the cerebral cortex. In Harlow, H.F. \& Woolsey, C.N. (eds), The Biological and Biochemical Bases of Behavior. University of Wisconsin, Madison.

Worsley, K.J. \& Friston, K.J. (1995) Analysis of fMRI time-series revisited again. Neuroimage, 3, 173-181. 